\title{
THE INTERPOLATION OF PICK FUNCTIONS
}

WILLIAM F. DONOGHUE, JR.

Before stating our version of the Cauchy Interpolation Problem it is desirable to recall the definition of the degree of a rational function. Let $f(z)$ be a rational function; then, in a known way, $f(z)$ may be regarded as a continuous map of the Riemann sphere into itself. This mapping has a Brouwer degree, $d$, which we take to be the degree of the rational $f(z)$. Equivalently, if $f(z)$ is presented as the quotient of two relatively prime polynomials $p(z)$ and $q(z)$, where $d^{\prime}$ is the algebraic degree of $p$ and $d^{\prime \prime}$ is the algebraic degree of $q$ then the degree of $f(z)$ is given by $d=\max \left(d^{\prime}, d^{\prime \prime}\right)$. Finally, we should note that for all but finitely many values of $\lambda$ the function $f(z)-\lambda$ has exactly $d$ distinct and finite zeros, and these are simple. If it is known that the rational $f(z)$ has degree at most $N$ and that it has at least $N+1$ zeros, multiplicitly included, then $f(z)$ vanishes identically.

Cauchy Interpolation Problem. Let there be given $k$ distinct interpolation points on the real axis $x_{1}, x_{2}, x_{3}, \cdots, x_{k}$ and equally many non-negative integers $\nu_{1}, \nu_{2}, \nu_{3}, \cdots, \nu_{k}$ as well as $N=\sum_{i=1}^{k}\left(\nu_{i}+1\right)$ real numbers $f_{i j}$ where $1 \leqq i \leqq k$ and $0 \leqq j \leqq \nu_{i}$. It is required to find a rational function $f(z)$ of degree at most $N / 2$ satisfying the $N$ conditions $f^{(j)}\left(x_{i}\right)=f_{i j}$. In any case that we study, the problem will in fact be an interpolation problem: there will be a function $F(z)$, usually not rational, so that the data $f_{i j}$ are obtained from $F^{(j)}\left(x_{i}\right)$.

In the special case when $N=k$, where no derivatives were considered in the problem, the Cauchy Interpolation Problem was exhaustively studied by Löwner in a famous paper [2]. The other extreme case, where $k=1$, corresponds to the determination of certain Padé approximations of a function, these approximations being on the diagonal or adjacent to the diagonal in the Padé table.

It is important to note that if $f(z)$ is a solution to the Cauchy Interpolation Problem for which the degree of $f(z)$ is strictly smaller than $N / 2$ then the solution is unique. Were there another solution $g(z)$, the rational function $f(z)-g(z)$ would have degree at most $N-1$, but would have at least $N$ zeros, since at each interpolation point $x_{i}$ there would be a zero of degree $\nu_{i}+1$. Thus the difference would vanish identically. We emphasize that this will always be the case when $N$ is odd. It is therefore clear that the Interpolation Problem depends significantly on the parity of $N$.

Received by editors February 8, 1973. 
In order to state the useful theorems concerning the Cauchy Interpolation Problem it is convenient to introduce the concept of the divided differences of a function. In view of the fact that we shall be concerned almost exclusively with analytic functions, we give a somewhat unorthodox definition. Let $F(z)$ be analytic in some region $D$ and suppose that $\lambda_{0}, \lambda_{1}, \lambda_{2}, \cdots, \lambda_{\ell}$ is a system of $\ell+1$ not necessarily distinct points in $D$. Let $C$ be a rectifiable curve in $D$ which surrounds these points. We then set

$\left[\lambda_{0}, \lambda_{1}, \lambda_{2}, \cdots, \lambda_{\ell}\right]=\frac{1}{2 \pi i} \int_{C} \frac{F(z)}{\left(z-\lambda_{0}\right)\left(z-\lambda_{1}\right)\left(z-\lambda_{2}\right) \cdots\left(z-\lambda_{\ell}\right)} d z$.

It is plain to see that this "divided difference" can be evaluated by residues and that it will be an algebraic expression in the points $\lambda_{i}$ and the values of the function $F$ at those points as well as the values of some of the derivatives. Indeed, if we take for the $\lambda$ the points of the Cauchy Interpolation Problem, each $x_{i}$ being taken $\nu_{i}+1$ times, then the resulting difference will involve exactly the data of the Cauchy Interpolation Problem. It becomes clear that we could have stated that problem in an equivalent way: all possible values of the differences $\left[\lambda_{0}, \lambda_{1}, \cdots, \lambda_{\ell}\right]$ are prescribed, whenever the set of $\lambda^{\prime}$ 's is a subset of the $N$ numbers

$$
\left[x_{1}, x_{1}, \cdots, x_{2}, x_{2}, \cdots, x_{3}, x_{3}, \cdots, x_{k}, x_{k}\right]
$$

where each $x_{i}$ is taken $\nu_{i}+1$ times. Then it is necessary to find a rational function $f(z)$ of degree at most $N / 2$ realizing these differences.

Let us suppose that $N$ is even and write $N=2 n$. Take the set of $N$ numbers corresponding to the Cauchy problem and divide these numbers into two sets of $n$ elements each in any way that is convenient. Call the elements of the first set $\xi_{1}, \xi_{2}, \cdots, \xi_{n}$ and those of the second $\eta_{1}, \eta_{2}, \cdots, \eta_{n}$. Now form the matrix of order $n: L=$

$\left[\begin{array}{ccc}{\left[\xi_{1}, \eta_{1}\right]} & {\left[\xi_{1}, \eta_{1}, \eta_{2}\right]} & {\left[\xi_{1}, \eta_{1}, \eta_{2}, \cdots, \eta_{n}\right]} \\ {\left[\xi_{1}, \xi_{2}, \eta_{1}\right]} & {\left[\xi_{1}, \xi_{2}, \eta_{1}, \eta_{2}\right]} & {\left[\xi_{1}, \xi_{2}, \eta_{1}, \eta_{2}, \cdots, \eta_{n}\right]} \\ {\left[\xi_{1}, \xi_{2}, \cdots, \xi_{n}, \eta_{1}\right]} & & {\left[\xi_{1}, \xi_{2}, \cdots, \xi_{n}, \eta_{1}, \eta_{2}, \cdots, \eta_{n}\right]}\end{array}\right]$

Note that the $i j$-th element of this matrix is $\left[\xi_{1}, \xi_{2}, \cdots, \xi_{i}, \eta_{1}, \eta_{2}, \cdots, \eta_{j}\right]$.

THEOREM 1. L will be singular or non-singular in a way that depends only on the data of the interpolation problem, and independently of the assignment of the names $\xi_{i}$ and $\eta_{j}$ in that problem. When $L$ is non-singular there exist two pairs of real polynomials 
$\left[\sigma_{0}, \tau_{0}\right]$ and $\left[\sigma_{\infty}, \tau_{\infty}\right]$ of degree at most $n$ such that all solutions to the Cauchy problem occur in the family

$$
f_{t}(z)=\frac{\sigma_{0}(z)+t \sigma_{\infty}(z)}{\tau_{0}(z)+t \tau_{\infty}(z)} .
$$

All functions in this family are solutions to the Interpolation Problem save for at most $k$ exceptional solutions where numerator and denominator have a common zero at an interpolation point. The corresponding rational function is then of degree strictly smaller than $n$ and is not a solution to the Interpolation Problem.

Our next theorem does not require that $N$ be even.

Theorem 2. A solution to the Cauchy Interpolation Problem exists if there exists an integer $n$ with $2 n \geqq \max \nu_{i}$ such that all $n$ by $n$ matrices $L$ of the type above formed from data of the problem are non-singular, while all such matrices of higher order are singular. The solution then is exactly of degree n, but it need not be unique.

\section{Pick Functions.}

A function $\varphi(\zeta)=U(\zeta)+i V(\zeta)$ is called a Pick function if it is analytic in the upper half-plane and has positive imaginary part. Such functions admit a canonical representation, easily derived from the Poisson integral representation of the harmonic and positive $V(\zeta)$. We will have

$$
\varphi(\zeta)=\alpha \zeta+\beta+\int\left[\frac{1}{\lambda-\zeta}-\frac{\lambda}{\lambda^{2}+1}\right] d \mu(\lambda)
$$

where $\alpha \geqq 0, \beta$ is real and $\mu$ a positive Radon measure on the real $\lambda$-axis for which $\int\left(\lambda^{2}+1\right)^{-1} d \mu(\lambda)$ is finite. The representation is unique, in fact, putting $\zeta=\xi+i \eta$ we have $\alpha=\lim _{\eta \rightarrow \infty} V(i \eta) / \eta$ and $\beta=\operatorname{Re}[\varphi(i)]$ while the measure $\mu$ may be determined from the function in the following way. We consider a monotone increasing function $\mu(\lambda)$ corresponding to the measure and normalize it so that $\boldsymbol{\mu}(\lambda)=(\boldsymbol{\mu}(\lambda+0)+\boldsymbol{\mu}(\lambda-0)) / 2$. Under these circumstances, then for every interval $(a, b)$

$$
\mu(b)-\mu(a)=\lim _{\eta \rightarrow 0} \frac{1}{\pi} \int_{a}^{b} V(x+i \eta) d x .
$$

Associated with the open interval $(a, b)$ we have the subclass of Pick functions denoted $P(a, b)$ : these are the Pick functions which admit an analytic continuation from the upper half-plane across the interval $(a, b)$ into the lower half-plane such that the continuation is 
by reflection with respect to the real axis. It is not difficult to show that $\varphi(\zeta)$ is in $P(a, b)$ if and only if the corresponding measure $\mu$ puts no mass in the interval $(a, b)$. A function $F(z)$ is a series of Stieltjes if and only if $-F(z)$ belongs to $P(0, \infty)$. If a rational function $\varphi(\zeta)$ belongs to $P(a, b)$ then its poles are simple, fall on the real axis outside the interval $(a, b)$ and have negative residues.

In this paper we will suppose that a Pick function $\varphi(\zeta)$ is given and that it belongs to the class $P(a, b)$; we seek to approximate the function with solutions to the Cauchy Interpolation Problem associated with the given function where the interpolation points $x_{i}$ are all within the open interval. It is easier to describe the situation if we suppose, in addition, that $\varphi$ is not a rational function.

Theorem 3. The square matrices L associated with the interpolation are non-singular.

While we avoid giving the proofs of our theorems, it is worthwhile to indicate its nature here. Consider the functions $f_{i}(\lambda)$ and $g_{j}(\lambda)$ where $f_{i}(\lambda)^{-1}=\left(\lambda-\xi_{1}\right)\left(\lambda-\xi_{2}\right) \cdots\left(\lambda-\xi_{i}\right)$ and $g_{j}(\lambda)^{-1}=$ $\left(\lambda-\eta_{1}\right)\left(\lambda-\eta_{2}\right) \cdots\left(\lambda-\eta_{j}\right)$. Using the canonical representation of $\varphi$, at least under the additional hypothesis that $\alpha=0$, we find

$$
\begin{aligned}
L_{i j}=\left[\xi_{1}, \xi_{2}, \cdots, \xi_{1}, \eta_{1}, \eta_{2}, \cdots, \eta_{j}\right] & =\int f_{i}(\lambda) g_{j}(\lambda) d \mu(\lambda) \\
& =\left(f_{i}, g_{j}\right)
\end{aligned}
$$

the inner product being taken in the $L^{2}$-space associated with the measure $\mu$. Thus the matrix $L_{i j}$ is a sort of Gram's matrix, and as in $[1]$, it is easy to show that it is non-singular when the support of the measure is not a finite set.

The important theorem is essentially due to Loewner [1,2].

Theorem 4. Let $N$ be odd and $\varphi_{N}$ the corresponding (unique) interpolation for $\varphi$; then $\varphi_{N}$ is also in $P(a, b)$. When $N$ is even many of the interpolating functions are in the Pick class, but not all.

In view of certain well-known compactness properties for the Pick class and the class $P(a, b)$ and because of the form of the approximating functions when $N$ is even, the successive approximations to $\varphi$ display the usual limit-point and limit circle behavior associated with the moment problem as well as with the Sturm-Liouville problem. However, in almost every case, only the limit-point case occurs and the approximating functions converge to $\varphi(\zeta)$ uniformly on compact subsets of the union of the upper half-plane, the lower half-plane and the interval $(a, b)$. The convergence is generally extremely rapid and the approximations oscillate about the limiting function in certain inter- 
vals. We refer to the paper of M. F. Barnsley in these proceedings for further detail.

\section{REFERENCES}

1. William F. Donoghue, Jr., The theorems of Loewner and Pick, Israel Journal of Mathematics 4 (1966), 153-170.

2. K. Löwner, Über Monotone Matrixfunktionen, Mathematische Zeitschrift 38 (1934), 177-216.

University of California, Irvine, California 92664 
